Z. Epileptol. 2022 · 35:29-31 https://doi.org/10.1007/s10309-021-00464-7 Angenommen: 22. Dezember 2021 Online publiziert: 14. Januar 2022

๑) Der/die Autor(en) 2022

\section{MEG bei frontalen Epilepsien}

Stefan Rampp ${ }^{1}$ Burkhard Kasper ${ }^{2}$. Sebastian Brandner ${ }^{1}$ Hajo Hamer ${ }^{2}$.

Michael Buchfelder'

${ }^{1}$ Neurochirurgische Klinik, Universitätsklinik Erlangen, Erlangen, Deutschland

${ }^{2}$ Epilepsiezentrum, Neurologische Universitätsklinik Erlangen, Erlangen, Deutschland

\title{
Zusammenfassung
}

Die Magnetenzephalographie (MEG) bietet für die prächirurgische Lokalisationsdiagnostik frontaler und allgemein extratemporaler Epilepsien wesentliche Vorteile. Eine Reihe retro- und prospektiver Studien zeigt einen signifikanten Einfluss auf die Planung invasiver Ableitungen und epilepsiechirurgischer Eingriffe mit deutlicher Verbesserung der postoperativen Anfallskontrolle. Der Artikel gibt einen kurz gefassten Überblick über den aktuellen Stand der Evidenz.

\section{Schlüsselwörter}

Epilepsiechirurgie · MEG · Lokalisation · Anfallskontrolle · Quellenanalyse

Ungefähr ein Drittel aller Patienten mit fokalen Epilepsien wird mit einer medikamentösen Therapie nicht anfallsfrei. Für Patienten mit solchen pharmakoresistenten fokalen Epilepsien stellt die Epilepsiechirurgie eine Therapieoption dar [22]. Je nach Ätiologie werden ca. $50-80 \%$ der Patienten anfallsfrei [10]. Zirka 25\% der operierten Patienten leiden unter einer Frontallappenepilepsie (FLE) [1]. Im Vergleich zu den Temporallappenepilepsien (TLE) sind die postoperativen Ergebnisse hier deutlich schlechter. Während ca. 70\% TLE-Patienten im Langzeitverlauf rezidivfrei bleiben, ist das bei Patienten mit FLE deutlich seltener der Fall $[5,7]$.

\section{Lokalisation der FLE}

Die Gründe für diese Diskrepanz liegen in der deutlich schwierigeren Lokalisation und vollständigen Resektion der epileptogenen Zone. Die Semiologie der FLE erlaubt allenfalls regionale Zuordnungen mit Ausnahme von Foci mit direkter Beziehung zu eloquenten Arealen [1]. Eben diese Lagebeziehungen können jedoch die Resektion einschränken und die postoperative Anfallskontrolle beeinträchtigen [7].

Das EEG kann bei FLE unspezifisch bleiben und bei Anfällen mit motorischer Komponente Artefakt-überlagert sein [1]. Die Interpretation wird zudem durch eine teilweise tangentiale Ausrichtung der zugrunde liegenden Neuronenpopulationen erschwert. Hier erscheint die maximale Negativität in Referenz- bzw. die Phasenumkehr in bipolaren Ableitungen nicht über der Quelle, sondern kann fern von ihr liegen [17]. Besonders bei mesialen Quellen kann dadurch die Seitenzuordnung verfälscht erscheinen. Kleine Änderungen in der Gesamtausrichtung, z. B. durch Ausbreitung in benachbarte Sulci, können leicht als Propagation oder bilaterale Ausbreitung fehlinterpretiert werden [1, 17]. Die ausgeprägte intra- und interlobäre Konnektivität der Frontallappen kann zudem zu einer schnellen tatsächlichen Propagation führen [3], die jedoch nicht regelhaft auftritt [2].

Schließlich hat der Nachweis bzw. das Fehlen einer epileptogenen Läsion einen wesentlichen Einfluss auf die postoperative Anfallskontrolle. Die FLE unterscheidet sich hier v. a. in der Art der Läsion von der TLE mit entsprechender Konsequenz für den Erfolg eines Eingriffs [10].

\section{MEG}

Die Magnetenzephalographie (MEG) bietet bei der Diagnostik extratemporaler Epilepsien (ETLE) und damit auch der FLE signifikante Vorteile [18]. MEG registriert das von neuronaler Aktivität generierte Mag- 


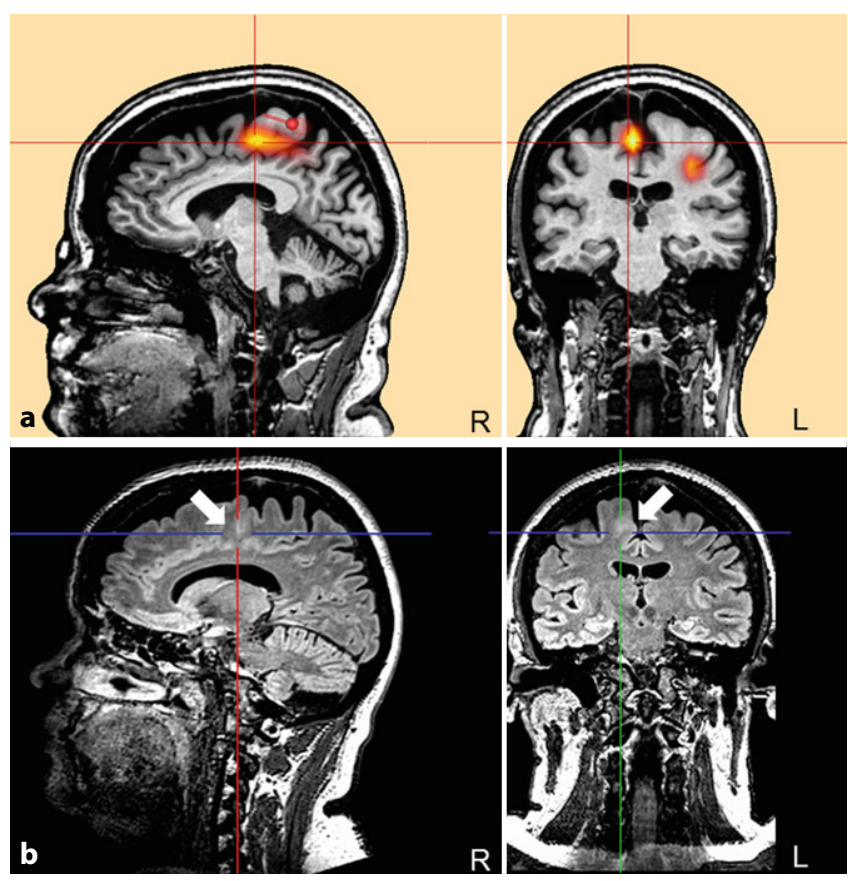

Abb. 14 MEG-

Lokalisation (a) mit sog. CLARA(gelb-rot, "Classical LORETA Recursively Applied", eine Lokalisationsmethode mittels verteilter Quellenaktivierung) und DipolVerfahren (rote Markierung) bei einer Patientin mit hochfrontaler, mesialer FCD 2b (Pfeile, b). Keine interiktalen epilepsietypischen Potenziale im EEG. Anfallsfrei (Engel 1a, Follow-up 3 Jahre) nach Resektion netfeld unter Abschirmung externer Störungen der Umgebung. Die Technik basiert auf quantenmechanischen Phänomenen bei Supraleitung, die eine Kühlung mit flüssigem Helium erfordert.

Die Sensitivität des MEG ist für neokortikale Quellen besonders hoch, wenn diese eine stark tangentiale Ausrichtung haben. Im EEG kann sich im Fall umschriebener neuronaler Populationen nur ein subtiles Korrelat zeigen oder fehlen [16], wobei die Anzahl der verwendeten EEG-Elektroden eine gewisse Rolle spielt [17].

Ein wesentlicher Vorteil ist die weitestgehende Unempfindlichkeit gegenüber Leitfähigkeitsunterschieden. Die Ausbreitung elektrischer Signale wird durch die unterschiedlichen elektrischen Leitfähigkeiten von Liquor, Knochen etc. signifikant beeinflusst. Bei großen Läsionen, Knochenlücken z.B. nach einer Operation, kortikalen Malformationen etc. können diese Verhältnisse verändert sein und müssen für eine präzise Lokalisation berücksichtigt werden - sei es bei der Quellenanalyse oder der visuellen Interpretation. Magnetische Leitfähigkeiten biologischer Gewebe sind nahezu identisch und spielen für die Volumenleitung hier keine wesentliche Rolle. MEG-Signale werden in solchen Fällen nicht verzerrt, und die Lokalisation wird erleichtert [17].

\section{MEG in der klinischen Anwendung}

Diese theoretischen Vorteile finden sich auch in der klinischen Anwendung der MEG-Quellenlokalisation wieder. Die - Abb. 1 zeigt ein Beispiel. In unserer retrospektiven Studie zum MEG in der prächirurgischen Diagnostik [18] war eine vollständige Resektion der MEG-Befunde bei TLE und ETLE signifikant mit Anfallsfreiheit auch nach 8 bis 10 Jahren verbunden; Patienten mit ETLE profitierten besonders. Weitere, auch prospektive Studien bestätigen sowohl die Rolle der vollständigen Resektion als auch den besonderen Beitrag bei ETLE bei Erwachsenen [4, 8, 13, 20, 21] und Kindern [6, 14].

In Mu et al. [12] untersuchten wir spezifisch 46 operierte Patienten mit FLE. Insgesamt $48 \%$ waren 5 Jahre nach dem Eingriff anfallsfrei. Monofokale Befunde, Lokalisation in der nichtdominanten Hemisphäre und eine komplette Resektion der MEG-Befunde waren signifikant mit einer postoperativen Anfallsfreiheit assoziiert. Die Ergebnisse von Wu et al. [23] illustrieren die komplementäre Sensitivität des MEG [16]. In der Studie wurden 30 FLEPatienten untersucht und Video-EEG-Monitoring mit MEG verglichen. Bei 6 von 20 Patienten mit Spikes im MEG fanden sich weder interiktal noch iktal epilepsietypische Muster im Video-EEG trotz der deut- lich längeren Ableitungszeit. Analog dazu berichten Ossenblok et al. [15] häufigere Spike-Detektionen im MEG in simultanen MEG/EEG-Ableitungen in 18 von 24 Patienten mit FLE. Die MEG-Analyse war zudem bei 14 Patienten erfolgreich im Vergleich zu 7 Patienten im EEG.

Eine herausfordernde Gruppe nicht nur bei vermuteter FLE oder ETLE sind schließlich Patienten mit unauffälligem MRT [22]. Mohamed et al. [9] zeigen in einer prospektiven Studie mit 57 Patienten (32 ETLE), dass MEG in ca. $21 \%$ die Planung einer invasiven Ableitung sowie des epilepsiechirurgischen Eingriffs signifikant verbessert hat. In einer früheren Studie [19] untersuchten wir die Kombination von MEG, Neuronavigation und intraoperatives MRT in einer Gruppe von 28 Patienten mit FLE. Patienten mit non-läsioneller FLE erreichten mit diesem multimodalen Ansatz Anfallsfreiheit in $64 \%$. Patienten mit klaren epileptogenen Läsionen zeigten mit $61 \%$ ein vergleichbares Ergebnis. Schließlich können MEGBefunde auf subtile Veränderungen hinweisen, die den sekundären Nachweis einer epileptogenen Läsion ermöglichen [11].

\section{Schlussfolgerung}

MEG bietet somit einen wesentlichen Beitrag zur prächirurgischen Diagnostik pharmakoresistenter Epilepsien. Patienten mit FLE profitieren durch den hohen diagnostischen Nutzen bei ETLE. Mit gegenwärtig ca. einem Dutzend über Deutschland verteilter Systeme ist die Technik grundsätzlich verfügbar. Ein wesentliches Hindernis für den breiteren Einsatz stellt jedoch die eingeschränkte Abrechenbarkeit dar.

\section{Fazit für die Praxis}

- MEG bietet eine hohe Sensitivität insbesondere für neokortikale und tangentiale Quellen.

- MEG wird durch Veränderungen der Anatomie nicht beeinträchtigt.

- Ein Einsatz ist besonders bei FLE und ETLE zur Planung invasiver Ableitungen und Epilepsiechirurgie sinnvoll.

- Insbesondere Patienten ohne klare EEGund MRT-Befunde können profitieren.

- Wesentliches Hindernis für den breiteren Einsatz ist die eingeschränkte Abrechenbarkeit. 
Korrespondenzadresse

PD Dr. med. Stefan Rampp

Neurochirurgische Klinik, Universitätsklinik

Erlangen

Schwabachanlage 6, 91054 Erlangen,

Deutschland

stefan.rampp@uk-erlangen.de

Funding. Open Access funding enabled and organized by Projekt DEAL.

\section{Einhaltung ethischer Richtlinien}

Interessenkonflikt. S. Rampp, B. Kasper, S. Brandner, H. Hamer und M. Buchfelder geben an, dass kein Interessenkonflikt besteht.

Für diesen Beitrag wurden von den Autoren keine Studien an Menschen oder Tieren durchgeführt. Für die aufgeführten Studien gelten die jeweils dort angegebenen ethischen Richtlinien.

Open Access. Dieser Artikel wird unter der Creative Commons Namensnennung 4.0 International Lizenz veröffentlicht, welche die Nutzung, Vervielfältigung, Bearbeitung, Verbreitung und Wiedergabe in jeglichem Medium und Format erlaubt, sofern Sie den/die ursprünglichen Autor(en) und die Quelle ordnungsgemäß nennen, einen Link zur Creative Commons Lizenz beifügen und angeben, ob Änderungen vorgenommen wurden.

Die in diesem Artikel enthaltenen Bilder und sonstiges Drittmaterial unterliegen ebenfalls der genannten Creative Commons Lizenz, sofern sich aus der Abbildungslegende nichts anderes ergibt. Sofern das betreffende Material nicht unter der genannten Creative Commons Lizenz steht und die betreffende Handlung nicht nach gesetzlichen Vorschriften erlaubt ist, ist für die oben aufgeführten Weiterverwendungen des Materials die Einwilligung des jeweiligen Rechteinhabers einzuholen.

Weitere Details zur Lizenz entnehmen Sie bitte der Lizenzinformation auf http://creativecommons.org/ licenses/by/4.0/deed.de.

\section{Literatur}

1. Beleza P, Pinho J (2011) Frontal lobe epilepsy. J Clin Neurosci 18:593-600. https://doi.org/10.1016/J. JOCN.2010.08.018

2. Blume WT, Ociepa D, Kander V (2001) Frontal lobe seizure propagation: scalp and subdural EEG studies. Epilepsia 42:491-503. https://doi.org/10. 1046/J.1528-1157.2001.26700.X

3. Bonini F, McGonigal A, Trébuchon A et al (2014) Frontal lobe seizures: from clinical semiology to localization. Epilepsia 55:264-277. https://doi. org/10.1111/EPI.12490

4. De Tiège X, Carrette E, Legros B et al (2012) Clinical added value of magnetic source imaging in the presurgical evaluation of refractory focal epilepsy. JNeurol Neurosurg Psychiatry 83:417-423. https:// doi.org/10.1136/jnnp-2011-301166

5. Englot DJ, Wang DD, Rolston JD et al (2012) Rates and predictors of long-term seizure freedom after

\section{MEG in frontal epilepsy}

Magnetoencephalography (MEG) offers considerable advantages for presurgical localization diagnostics of frontal and generalized extratemporal epilepsy. A number of retrospective and prospective studies show a significant impact on the planning of invasive recordings and epilepsy surgical procedures with marked improvement of postoperative seizure control. This article provides a concise overview of the current state of the evidence.

\section{Keywords}

Epilepsy surgery $\cdot$ MEG $\cdot$ Localization · Seizure control $\cdot$ Source analysis frontal lobe epilepsy surgery: a systematic review and meta-analysis. J Neurosurg 116:1042-1048. https://doi.org/10.3171/2012.1.JNS111620

6. Gautham B, Ahmed A, Mundlamuri RC et al (2021) Magnetic source imaging in presurgical evaluation of paediatric focal drug-resistant epilepsy and its predictive value of surgical outcome in lesional cases: A single-centre experience from South India. Seizure 91:22-28. https://doi.org/10.1016/j. seizure.2021.05.015

7. Jeha LE, Najm I, Bingaman W et al (2007) Surgical outcome and prognostic factors of frontal lobe epilepsy surgery. Brain 130:574-584. https://doi. org/10.1093/BRAIN/AWL364

8. Kasper BS, Rössler K, Hamer HM et al (2018) Coregistrating magnetic source and magnetic resonance imaging for epilepsy surgery in focal cortical dysplasia. Neuroimage Clin 19:487-496. https://doi.org/10.1016/j.nicl.2018.04.034

9. Mohamed IS, Toffa DH, Robert M et al (2020) Utility of magnetic source imaging in nonlesional focal epilepsy: a prospective study. Neurosurg Focus 48:E16. https://doi.org/10.3171/2020.1. FOCUS19877

10. Mohan M, Keller S, Nicolson A et al (2018) The long-term outcomes of epilepsy surgery. PLoS ONE 13:e196274. https://doi.org/10.1371/journal. pone. 0196274

11. Moore KR, Funke ME, Constantino T et al (2002) Magnetoencephalographically directed review of high-spatial-resolution surface-coil MR images improves lesion detection in patients with extratemporal epilepsy. Radiology 225:880-887. https://doi.org/10.1148/radiol.2253011597

12. Mu J, Rampp S, Carrette E et al (2014) Clinical relevance of source location in frontal lobe epilepsy and prediction of postoperative longterm outcome. Seizure 23:553-559. https://doi. org/10.1016/j.seizure.2014.04.006

13. Murakami H, Wang Zl, Marashly A et al (2016) Correlating magnetoencephalography to stereoelectroencephalography in patients undergoing epilepsy surgery. Brain 139:2935-2947. https:// doi.org/10.1093/brain/aww215

14. Ntolkeras G, Tamilia E, AlHilani M et al (2021) Presurgical accuracy of dipole clustering in MRI-negative pediatric patients with epilepsy: Validation against intracranial EEG and resection. Clin Neurophysiol. https://doi.org/10.1016/J. CLINPH.2021.01.036

15. Ossenblok P, de Munck JC, Colon A et al (2007) Magnetoencephalography is more successful for screening and localizing frontal lobe epilepsy than electroencephalography. Epilepsia 48:2139-2149. https://doi.org/10.1111/j.1528-1167.2007.01223. $\mathrm{x}$
16. Piastra MC, Nüßing A, Vorwerk J et al (2020) A comprehensive study on electroencephalography and magnetoencephalography sensitivity to cortical and subcortical sources. Hum Brain Mapp. https://doi.org/10.1002/hbm.25272

17. Rampp S (2018) EEG- und MEG-Quellenlokalisation. Z Epileptol 31:109-112. https://doi.org/10 1007/s10309-017-0164-8

18. Rampp S, Stefan H, Wu X et al (2019) Magnetoencephalography for epileptic focus localization in a series of 1000 cases. Brain 142:3059-3071. https://doi.org/10.1093/brain/awz231

19. Sommer B, Roessler K, Rampp S et al (2016) Magnetoencephalography-guided surgery in frontal lobe epilepsy using neuronavigation and intraoperative MR imaging. Epilepsy Res 126:26-36. https://doi.org/10.1016/j.eplepsyres. 2016.06.002

20. Tripathi M, Kaur K, Ramanujam B et al (2021) Diagnostic added value of interictal magnetic source imaging in presurgical evaluation of persons with epilepsy: a prospective blinded study. Eur J Neurol 28:2940-2951. https://doi.org/ 10.1111/ene.14935

21. Vadera S, Jehi L, Burgess RC et al (2013) Correlation between magnetoencephalographybased "clusterectomy" and postoperative seizure freedom. Neurosurg Focus 34:E9. https://doi.org/ 10.3171/2013.4.FOCUS1357

22. West S, Nolan SJ, Cotton J et al (2015) Surgery for epilepsy. Cochrane Database Syst Rev. https://doi. org/10.1002/14651858.CD010541.pub2

23. Wu X-T, Rampp S, Hopfengärtner R et al (2012) Complementary use of video-electroencephalography and magnetoencephalography in frontal lobeepilepsy. Seizure 21:426-430.https://doi.org/ 10.1016/j.seizure.2012.04.007 